\title{
A Class of Meromorphic p-valent Functions
}

\author{
Waggas Galib Atshan \\ Sarah Abd Al-Hmeed Jawad \\ Department of Mathematics, College of Computer Science and Information \\ Technology, University of Qadisiayh,Diwaniyah, \\ waggas.galib@qu.edu.iq Sarahabdalhmeed94@gmail.com
}

\begin{abstract}
Available online : $\quad 28 / 1 / 2019$
\end{abstract}
DOI: $10.29304 / j q c m .2019 .11 .1 .479$

\begin{abstract}
:
In this paper, we define a class of meromorphic p-valent functions and study some properties as coefficient inequality, closure theorem, growth and distortion bounds, arithmetic mean, radius of convexity, Convex linear combination and partial sums .
\end{abstract}

Keywords: Meromorphic p-valent function,Convex function, Integral operator .

Mathematics Subject Classification: 30C45. 
1-Introduction: Let $A_{p}^{*}$ denote the class of functions $f$ of the form

$f(z)=z^{-p}+\sum_{n=p}^{\infty} a_{n} z^{n},\left(a_{n} \geq 0, n \geq p, p \in N\right)$, (1.1)

which are analytic and p-valent in the punctured unit disk $U^{*}=\{\mathrm{z} \in \mathrm{C}: 0<|z|<1\}$. Jum-Kim Srivastara [2] defined an integral operator $I_{p}^{\sigma} f(z)$ for $f \in A_{p}^{*}$ as follows

$I_{p}^{\sigma} f(z)=\frac{1}{z^{p+1} \Gamma(\sigma)} \int_{0}^{z}\left(\log \frac{z}{t}\right)^{\sigma-1} t^{p} f(z) d t,(n \in N)$. (1.2)

If $f(z)$ is of the form (1.1), then

$I_{p}^{\sigma} f(z)=z^{-p}+\sum_{n=p}^{\infty}\left(\frac{1}{n+p+1}\right)^{\sigma} a_{n} z^{n}(n \geq p, p$

$$
\in N) \text {. }
$$

In particular, when $\mathrm{p}=1$ we have:

$I_{p}^{\sigma} f(z)=z^{-1}+\sum_{n=1}^{\infty}\left(\frac{1}{n+2}\right)^{\sigma} a_{n} z^{n}(n \geq p, p \in N)$.

Let $f$ and $g$ be analytic in unit disk $\mathrm{U}$, then $g$ is said to be subordinate of $f$, written as $g \prec f$ or $g(\mathrm{z}) \prec$ $f(\mathrm{z})$, if there exists a schwartz function $\omega$ which is analytic in $U$ with $\omega(0)=0$ and $|\omega(z)|<1(z \in U)$ such that $g(\mathrm{z})=f(\omega(\mathrm{z}))$.

In particular, if the function $\mathrm{f}$ is univalent in $\mathrm{U}$, we have the following equivalence ([3],[4]).

$$
\begin{gathered}
g(z) \prec f(z)(z \in U) \Leftrightarrow g(0)=f(0) \text { and } g(U) \\
\subseteq f(U) .
\end{gathered}
$$

Definition(1.1): A function $f \in A_{p}^{*}$ is said to be in the class $A_{p}^{*}(\sigma, b, x, y)$ of functions of the form (1.1), which satisfies the condition

$p-\frac{1}{b}\left\{1+\frac{z^{2}\left(I_{p}^{\sigma} f(z)\right)^{\prime \prime}}{z\left(I_{p}^{\sigma} f(z)\right)^{\prime}}+p\right\}$

$\prec p \frac{1+x z}{1+y z}$,

where

$-1 \leq y \leq x \leq 1, p \in N, \sigma<$

$0, b$ non zero complex number.

We can re-write the condition (1.4) as

$\left|\frac{z\left(I_{p}^{\sigma} f(z)\right)^{\prime \prime}+(1+p)\left(I_{p}^{\sigma} f(z)\right)^{\prime}}{y z\left(I_{p}^{\sigma} f(z)\right)^{\prime \prime}+[y(1+p(1-b))+x b p]\left(I_{p}^{\sigma} f(z)\right)^{\prime}}\right|<1$

\section{Coefficient inequality:}

In the following theorem, we give a sufficient and necessary condition to be the function in the class $A_{p}^{*}(\sigma, b, x, y)$.
Theorem (2.1): Let $f \in A_{p}^{*}$ be given by (1.1). Then $f \in A_{p}^{*}(\sigma, b, x, y)$ if and only if

$$
\begin{aligned}
\sum_{n=p}^{\infty}[n(n+p)(1 & -y) \\
& \left.-n p|b|\left(\begin{array}{c}
x \\
-y
\end{array}\right)\right]\left(\frac{1}{n+p+1}\right)^{\sigma} a_{n} \\
& \leq p^{2}|b|(x-y) .
\end{aligned}
$$

The results is sharp for the function $f$ given by $f(z)=z^{-p}+\left(\frac{p^{2}|b|(x-y)}{[n(n+p)(1-y)-n p|b|(x-y)]}\right)(n+p+$ 1) ${ }^{\sigma} z^{n},(n \geq p, n \in N)$. (2.2)

Proof: Assuming that the inequality (2.1) holds true and $|z|=1$. Then, we have

$$
\begin{aligned}
& \mid z^{2}\left(I_{p}^{\sigma} f(z)^{\prime \prime}+\right.(1+p) z\left(I_{p}^{\sigma} f(z)\right)^{\prime} \mid \\
&-\mid y z^{2}\left(I_{p}^{\sigma} f(z)\right)^{\prime \prime}+[y(1 \\
&+p(1-b))+x b p] z\left(I_{p}^{\sigma} f(z)\right)^{\prime} \mid \\
&=\left|\sum_{n=p}^{\infty} n(n+p)\left(\frac{1}{n+p+1}\right)^{\sigma} a_{n} z^{n}\right|-\left|p^{2}\right| b \mid(x-y)+ \\
& \sum_{n=p}^{\infty}[y n(n+p)+n|b| p(x-y)]\left(\frac{1}{n+p+1}\right)^{\sigma} a_{n} z^{n} \mid \\
& \leq \quad \sum_{n=p}^{\infty} n(n+p)\left(\frac{1}{n+p+1}\right)^{\sigma} a_{n}|z|^{n}-p^{2}|b|(x- \\
&y)-\quad \\
& \sum_{n=p}^{\infty}[y n(n+p)+n|b| p(x-y)]\left(\frac{1}{n+p+1}\right)^{\sigma} a_{n}|z|^{n} \\
&=\sum_{n=p}^{\infty} n(n+p)\left(\frac{1}{n+p+1}\right)^{\sigma} a_{n}-p^{2}|b|(x-y)- \\
& \sum_{n=p}^{\infty}[y n(n+p)+n|b| p(x-y)]\left(\frac{1}{n+p+1}\right)^{\sigma} a_{n} \leq 0,
\end{aligned}
$$

by hypothesis.

Hence, by the Maximum Modulus Theorem, we have $f(z) \in A_{p}^{*}(\sigma, b, x, y)$.

Conversely, suppose that $f(z) \in A_{p}^{*}(\sigma, b, x, y)$. Then from (1.5), we have

$$
\begin{aligned}
& \left|\begin{array}{c}
\frac{z^{2}\left(I_{p}^{\sigma} f(z)^{\prime \prime}+(1+p) z\left(I_{p}^{\sigma} f(z)\right)^{\prime}\right.}{y z^{2}\left(I_{p}^{\sigma} f(z)\right)^{\prime \prime}+[y(1+p(1-b))+x b p]} \\
z\left(I_{p}^{\sigma} f(z)\right)^{\prime}
\end{array}\right| \\
& \frac{\sum_{n=p}^{\infty} n(n+p)\left(\frac{1}{n+p+1}\right)^{\sigma} a_{n} z^{n}}{[y n(n+p)+n|b| p(x-y)]} \\
& p^{2}|b|(x-y)+\sum_{n=p}^{\infty}\left(\frac{1}{n+p+1}\right)^{\sigma} a_{n} z^{n}
\end{aligned} \mid
$$

$<1$.

Since $\operatorname{Re}(\mathrm{z}) \leq|z|$ for all $\mathrm{z}(\mathrm{z} \in U)$, we have $\operatorname{Re}($

$\left.\frac{\sum_{n=p}^{\infty} n(n+p)\left(\frac{1}{n+p+1}\right)^{\sigma} a_{n} z^{n}}{p^{2}|b|(x-y)+\sum_{n=p}^{\infty}[y n(n+p)+n|b| p(x-y)]\left(\frac{1}{n+p+1}\right)^{\sigma} a_{n} z^{n}}\right) \leq$

1. 
We choose the value of $\mathrm{z}$ on the real and $\mathrm{z} \rightarrow 1^{-}$, we get

$$
\begin{gathered}
\sum_{n=p}^{\infty}\left[n(n+p)(1-y)-n p|b|\left(\begin{array}{c}
x \\
-y
\end{array}\right)\right]\left(\frac{1}{n+p+1}\right)^{\sigma} a_{n} \\
\leq p^{2}|b|(x-y)
\end{gathered}
$$

which give (2.1).Sharpness of the result follows by setting

$f(z)=z^{-p}+\left(\frac{p^{2}|b|(x-y)}{[n(n+p)(1-y)-n p|b|(x-y)]}\right)(n+p+$ 1) ${ }^{\sigma} Z^{n},(n \geq p, n \in N)$.

Corollary (2.1) :Let $f(z) \in A_{p}^{*}(\sigma, b, x, y)$.Then $a_{n} \leq \frac{p^{2}|b|(x-y)}{[n(n+p)(1-y)-n p|b|(x-y)]\left(\frac{1}{n+p+1}\right)^{\sigma}} \quad,(n \geq p, n \in$ $N)$.

\section{Growth and the Distortion Bounds:}

In the following theorems, we obtain the growth and the distortion theorems for the function in the class $A_{p}^{*}(\sigma, b, x, y)$.

Theorem (3.1): If the function $f(z)$ defined by (1.1) is in the class $A_{p}^{*}(\sigma, b, x, y)$, then for $0<|z|=$ $r<1$, we have:

$r^{-p}-\left(\frac{|b|(x-y)(2 p+1)^{\sigma}}{2(1-y)-|b|(x-y)}\right) r^{p} \leq|f(z)|$

$\leq r^{-p}+\left(\frac{|b|(x-y)(2 p+1)^{\sigma}}{2(1-y)-|b|(x-y)}\right) r^{p}$,

where equality holds true for the function

$=z^{-p}+\left(\frac{|b|(x-y)(2 p+1)^{\sigma}}{2(1-y)-|b|(x-y)}\right) z^{p}$.

Proof: Since $f(z) \in A_{p}^{*}(\sigma, b, x, y)$.Then from (2.1)

$2 p^{2}(1-y)-p^{2}|b|(x-y)\left(\frac{1}{2 p+1}\right)^{\sigma} \sum_{n=p}^{\infty}\left|a_{n}\right| \leq$

$\sum_{n=p}^{\infty}[n(n+p)(1-y)-$

$p|b|(x-y)]\left(\frac{1}{n+p+1}\right)^{\sigma} a_{n} \leq p^{2}|b|(x-y)$,

we conclude that

$\sum_{n=p}^{\infty}\left|a_{n}\right| \leq \frac{|b|(x-y)(2 p+1)^{\sigma}}{2(1-y)-|b|(x-y)}$

Thus for $0<|z|=r<1$,

$$
\begin{aligned}
|f(z)| \leq|z|^{-p}+ & \sum_{n=p}^{\infty} a_{n}|z|^{n} \\
& \leq r^{-p}+r^{p} \sum_{n=p}^{\infty} a_{n}
\end{aligned}
$$

or

$|f(z)| \leq r^{-p}-\left(\frac{|b|(x-y)(2 p+1)^{\sigma}}{2(1-y)-|b|(x-y)}\right) r^{p}$, $|f(z)| \geq|z|^{-p}-\sum_{n=p}^{\infty} a_{n}|z|^{n} \geq r^{-p}-r^{p} \sum_{n=p}^{\infty} a_{n}$

or

$|f(z)| \geq r^{-p}-\left(\frac{|b|(x-y)(2 p+1)^{\sigma}}{2(1-y)-|b|(x-y)]}\right) r^{p}$.

On using (3.4) and (3.5) inequality (3.1) follows.

Theorem (3.2): If $f \in A_{p}^{*}(\sigma, b, x, y)$ then

$$
\begin{aligned}
& r^{-(p+1)}-\left(\frac{p|b|(x-y)(2 p+1)^{\sigma}}{2(1-y)-p^{2}|b|(x-y)}\right) r^{p-1} \\
& \leq\left|f(z)^{\prime}\right| \\
& \leq r^{-(p+1)}+\left(\frac{p|b|(x-y)(2 p+1)^{\sigma}}{2(1-y)-p^{2}|b|(x-y)}\right) r^{p-1} .
\end{aligned}
$$

The result is sharp for the function $f$ is given by (1.3)

Proof: The proof is similar to that of Theorem (3.1).

\section{Extreme Points}

In the next theorems, we obtain extreme points for the class $A_{p}^{*}(\sigma, b, x, y)$.

Theorem (4.1): Let $f_{p-1}(z)=z^{-p}$ and $f_{n}(z)=$ $z^{-p}+\left(\frac{p^{2}|b|(x-y)(n+p+1)^{\sigma}}{[n(n+p)(1-y)-n p|b|(x-y)]}\right) z^{n}$,

for $n \geq p$. Then $f(z) \in A_{p}^{*}(\sigma, b, x, y)$ if and only if it can be expressed in the form

$$
\begin{gathered}
f(z)=\sum_{n=p-1}^{\infty} \mu_{n} f_{n}(z), \text { where } \mu_{n} \\
\quad \geq 0 \text { and } \sum_{n=p-1}^{\infty} \mu_{n}=1 .
\end{gathered}
$$

Proof: Let

$$
\begin{gathered}
f(z)=\sum_{n=p-1}^{\infty} \mu_{n} f_{n}(z)=z^{-p}+ \\
\sum_{n=p}^{\infty}\left(\frac{p^{2}|b|(x-y)(n+p+1)^{\sigma} \mu_{n}}{[n(n+p)(1-y)-n p|b|(x-y)]}\right) z^{n} .
\end{gathered}
$$

Then

$$
[n(n+p)(1-y)-n p|b|(x-y)]
$$

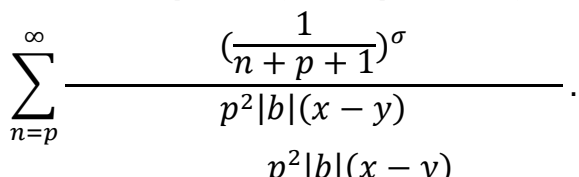

$\frac{p^{2}|b|(x-y)}{[n(n+p)(1-y)-n p|b|(x-y)]\left(\frac{1}{n+p+1}\right)^{\sigma}}$

$=\sum_{n=p}^{\infty} \mu_{n}=1-\mu_{p-1} \leq 1$.

Using Theorem (2.1),we easily get $f(z) \in$ $A_{p}^{*}(\sigma, b, x, y)$.

Conversely, let $(z) \in A_{p}^{*}(\sigma, b, x, y)$.

and 
From the Theorem (2.1), we have

$a_{n} \leq \frac{p^{2}|b|(x-y)(n+p+1)^{\sigma}}{[n(n+p)(1-y)-n p|b|(x-y)]}$ for $n$ $\geq p$.

Setting

$\mu_{n}=\frac{n(n+p)(1-y)-n p|b|(x-y)}{p^{2}|b|(x-y)}$.

$\left(\frac{1}{n+p+1}\right)^{\sigma}$ for $n \geq p$,

and $\mu_{p-1}=1-\sum_{n=p}^{\infty} \mu_{n}$.

Then

$f(z)=z^{-p}+\sum_{n=p}^{\infty} a_{n} z^{n}=$

$z^{-p}+\sum_{n=p}^{\infty}\left(\frac{p^{2}|b|(x-y)(n+p+1)^{\sigma} \mu_{n}}{[n(n+p)(1-y)-n p|b|(x-y)]}\right) z^{n}=$

$\mu_{p-1} z^{-p}+\sum_{n=p}^{\infty} \mu_{n} f_{n}(z)=\sum_{n=p-1}^{\infty} \mu_{n} f_{n}(z)$

This completes the proof.

\section{Radius of convexity}

In the following theorem, we obtain the radius of convexity for the function in the class $A_{p}^{*}(\sigma, b, x, y)$.

Theorem (5.1): Let $f$ the function $f(\mathrm{z})$ defined by (1.1) is in the class $A_{p}^{*}(\sigma, b, x, y)$. Then $f$ is meromorphically p-valent convex of order $\lambda(0 \leq$ $\lambda<p)$ in the disk $|z|<r_{2}$, where $r_{2}=$ $r_{2}(p, \sigma, b, x, y)=$

$\inf _{n \geq p}\left[\frac{(p-\lambda)[(n+p)(1-y)-p|b|(x-y)]\left(\frac{1}{n+p+1}\right)^{\sigma}}{(n+2 p-\lambda) p|b|(x-y)}\right]^{\frac{1}{n+p}}$

The result is sharp for the function $f$ given by (3.4).

Proof: A function $f$ meromorphic p-valent convex of order $\lambda(0 \leq \lambda<p)$ if

We must show that

$$
-\operatorname{Re}\left\{1+\frac{f^{\prime \prime}(z)}{f^{\prime}(z)}\right\}>\lambda \text {. }
$$

$\left|\frac{z f^{\prime \prime}(z)}{f^{\prime}(z)}+(1+p)\right|<p-\lambda, \quad$ for $\quad|z|<r_{2}$.

(5.2)

We have $\left|\frac{z f^{\prime \prime}(z)}{f^{\prime}(z)}+(1+p)\right|=\left|\frac{z f^{\prime \prime}(z)+(1+p) f^{\prime}(z)}{f^{\prime}(z)}\right|=$ $\left|\frac{\sum_{n=p}^{\infty} n(n+p) a_{n} z^{n+p}}{-p+\sum_{n=p}^{\infty} n a_{n} z^{n+1}}\right| \leq \frac{\sum_{n=p}^{\infty} n(n+p) a_{n}|z|^{n+p}}{p-\sum_{n=p}^{\infty} n a_{n}|z|^{n+p}}$.

Thus ,(5.2) will be satisfied if

$\sum_{n=p}^{\infty} \frac{n(n+2 p-\lambda)}{p(p-\lambda)} a_{n}|z|^{n+p} \leq 1$.

(5.3)

Since $f \in A_{p}^{*}(\sigma, b, x, y)$, we have

$\sum_{n=p}^{\infty} \frac{[n(n+p)(1-y)-n p|b|(x-y)]\left(\frac{1}{n+p+1}\right)^{\sigma}}{p^{2}|b|(x-y)} a_{n}$

$\leq 1$.
Hence,(5.3) will be true if

$\frac{n(n+2 p-\lambda)}{p(p-\lambda)}|z|^{n+p} \leq\left[\frac{n(n+p)(1-y)-n p|b|(x-y)\left(\frac{1}{n+p+1}\right)^{\sigma}}{p^{2}|b|(x-y)}\right]$, or equivalently

$|z|$

$$
\begin{aligned}
& (p-\lambda)(n+p)(1-y)-n p|b|(x-y) \\
\leq & \left(\frac{\left(\frac{1}{n+p+1}\right)^{\sigma}}{(n+2 p-\lambda) p^{2}|b|(x-y)}\right)^{\frac{1}{n+p}}, n
\end{aligned}
$$

$\geq p$

which follows the result.

\section{Convex linear combination:}

Theorem (6.1): The class $A_{p}^{*}(\sigma, b, x, y)$ is closed under convex linear combinations.

Proof: Let $f_{1}$ and $f_{2}$ be the chance elements of $A_{p}^{*}(\sigma, b, x, y)$. Then for each $\mathrm{t}(0<t<1)$ plus $\left(a_{n}, b_{n} \geq 0\right)$. we show that $(1-t) f_{1}+t f_{2} \in$ $A_{p}^{*}(\sigma, b, x, y)$. Thus we have

$(1-t) f_{1}+t f_{2}=z^{-p}+\sum_{n=p}^{\infty}\left[(1-t) a_{n}+\right.$ $\left.t b_{n}\right] z^{n}$.

Hence

$\sum_{n=p}^{\infty}[n(n+p)(1-y)-$

$n p|b|(x-y)]\left(\frac{1}{n+p+1}\right)^{\sigma}\left[(1-t) a_{n}+t b_{n}\right]$.

$=(1-t) \sum_{n=p}^{\infty}[n(n+p)(1-y)$

$\left.-n p|b|\left(\begin{array}{c}x \\ -y\end{array}\right)\right]\left(\frac{1}{n+p+1}\right)^{\sigma} a_{n}$

$+t \sum_{n=p}^{\infty}-n p|b|(x-y)\left(\frac{1}{n+p+1}\right)^{\sigma} b_{n}$

$\leq(1-t) p^{2}|b|(x-y)+t p^{2}|b|(x-y)$

$=p^{2}|b|(x-y)$.

This completes the proof.

\section{The anthmetic mean:}

Theorem (7.1): Let the functions $f_{k}$ sharp by $f_{k}(z)=z^{-p}+\sum_{n=p}^{\infty} a_{n, k},\left(a_{n, k} \geq 0, n \in N, k=\right.$ $1,2, \ldots l)$,

be in the class $A_{p}^{*}(\sigma, b, x, y)$ for each $\mathrm{k}=$ $(1,2,3, \ldots l)$, then the function $h$ sharp by

$$
h(z)=z^{-p}+\sum_{n=p}^{\infty} e_{n} z^{n},\left(e_{n} \geq 0, n \in N\right)
$$

also belong to the class $A_{p}^{*}(\sigma, b, x, y)$, where $e_{n}=\frac{1}{l} \sum_{n=p}^{\infty} a_{n, k},(n \geq p, p \in N)$. 
proof: $\operatorname{As} f_{k} \in A_{p}^{*}(\sigma, b, x, y)$, it follows the Theorem (2.1) that

$$
\begin{aligned}
& \sum_{n=p}^{\infty}[n(n+p)(1-y)-n p|b|(x- \\
& y)]\left(\frac{1}{n+p+1}\right)^{\sigma} a_{n, k} \leq p^{2}|b|(x-y),
\end{aligned}
$$

for each $\mathrm{k}=1,2,3, \ldots . l$ Hence

$\sum_{n=p}^{\infty}[n(n+p)(1-y)$

$$
\begin{aligned}
& -n p|b|(x-y)]\left(\frac{1}{n+p+1}\right)^{\sigma} e_{n} \\
& =\sum_{n=p}^{\infty}[n(n+p)(1-y) \\
& -n p|b|(x \\
& -y)]\left(\frac{1}{n+p+1}\right)^{\sigma}\left(\frac{1}{l} \sum_{k=1}^{l} a_{n, k}\right) \\
& =\frac{1}{l} \sum_{k=1}^{l}\left(\sum_{n=p}^{\infty}[n(n+p)(1-y)\right. \\
& -n p|b|(x \\
& \left.-y)]\left(\frac{1}{n+p+1}\right) a_{n, k}\right) \\
& \leq \frac{1}{l} \sum_{k=1}^{l} p^{2}|b|(x-y) \\
& =p^{2}|b|(x-y) .
\end{aligned}
$$

Then $h \in A_{p}^{*}(\sigma, b, x, y)$.

\section{Partial sums}

Theorem(8.1): Let $f \in A_{p}^{*}(\sigma, b, x, y)$ be assumed by(1.1)and $g \in A_{p}^{*}(\sigma, b, x, y)$ be assumed by

$$
g(z)=z^{-p}+\sum_{n=p}^{\infty} b_{n} z^{n} .
$$

We define the partial sums $S_{1}(z)$ and $S_{k}(z)$ as follows :

$$
\begin{aligned}
& S_{1}(z)=z^{-1} \text { and } S_{k}(z)= \\
& z^{-p}+ \\
& \sum_{n=p}^{k-1} a_{n} z^{n},(k \in N \mid\{1\}) .
\end{aligned}
$$

Also suppose that

$\sum_{n=p}^{\infty} c_{n} a_{n} \leq 1, c_{n}=$

$$
\frac{[n(n+p)(1-y)-n p|b|(x-y)]\left(\frac{1}{n+p+1}\right)^{\sigma}}{p^{2}|b|(x-y)} \text {. }
$$

Then, we $\operatorname{hav} R e\left\{\frac{f(z)}{s_{k}(z)}\right\}>1-\frac{1}{c_{k}},(z \in U, k \in$

$N)$,

and Re $\left\{\frac{s_{k}(z)}{f(z)}\right\}>\frac{c_{k}}{1+c_{k}},(z \in U, k \in N)$. (8.4)

Each of the bounds in (8.3) and (8.4) is the best possible for $k \in N$.
Proof: We can see from (8.2) that $c_{n+1}>c_{n}>$ $1, n=p, p+1, p+2, p+3, \ldots$

Therefore, we have:

$$
\sum_{n=p}^{k=1} a_{n}+c_{k} \sum_{n=k}^{\infty} a_{n} \leq \sum_{n=p}^{\infty} c_{n} a_{n} \leq 1 .
$$

By setting

$$
\begin{aligned}
& g_{1}(z)=c_{k}\left[\frac{f(z)}{S_{k}(z)}-\left(1-\frac{1}{c_{k}}\right)\right] \\
& =1+\frac{c_{k} \sum_{n=k}^{\infty} a_{n} z^{n+1}}{1+\sum_{n=p}^{k-1} a_{n} z^{n+1}},
\end{aligned}
$$

and applying (8.5) we find that

$$
\begin{aligned}
& \left|\frac{g_{1}(z)-1}{g_{1}(z)+1}\right| \leq \\
& \frac{c_{k} \sum_{n=k}^{\infty} a_{n}}{2-2 \sum_{n=p}^{k-1} a_{n}-c_{k} \sum_{n=k}^{\infty} a_{n}} \text {, }
\end{aligned}
$$

which readily yields the assertion (8.3) if, we take

$$
f(z)=z^{-p}-\frac{z^{k}}{c_{k}} \text {. }
$$

Then

$\frac{f(z)}{s_{k}(z)}=1-\frac{z^{k}}{c_{k}} \rightarrow 1-\frac{1}{c_{k}}\left(z \rightarrow 1^{-}\right)$, which shows that the bound in (8.3) is the best possible for $k \in N$. Similarly, if we put

$$
\begin{aligned}
& \mathrm{g}_{2}(z)=\left(1+c_{k}\right)\left[\frac{\mathrm{S}_{k}(z)}{f(z)}-\frac{c_{k}}{1+c_{k}}\right]=1- \\
& \frac{\left(1+c_{k}\right) \sum_{n=k}^{\infty} a_{n} z^{n+1}}{1+\sum_{n=p}^{k-1} a_{n} z^{k+1}}
\end{aligned}
$$

and make use of (8.9), we have

$$
\begin{aligned}
& \left|\frac{g_{2}(z)-1}{g_{2}(z)+1}\right| \\
& \leq \frac{(1+c) \sum_{n=k}^{\infty} a_{n}}{2-2 \sum_{n=p}^{k-1} a_{n}+\left(1-c_{k}\right) \sum_{n=k}^{\infty} a_{n}},
\end{aligned}
$$

which leads us to the assertion (8.4). The bound (8.5) is sharp for each $k \in N$ with the function given by (6.7). The proof of the theorem is complete.

\section{References}

[1] A.W. Goodman, univalent functions and nonanalytic curves, Proc. Amer. Math. Soc. , 8(1975),598-601.

[2] I .B. Jung, Y.C. Kim and H. M Srivastava, The Hardy space of analytic functions associated with certain one parameter families of integral operations , J. Math.Anal Appl.,176(1993),138197.

[3] S.Owa, and H. M. Srivastava, Univalent and starlike generalized hypergeometric function ,Cand.J.Math.,37(5) (1987),1057-1077.

[4] S .Ruscheweyh, Neighbourhood of univalent function , Proc. Amer. Math. Soc., 81(1981),521-527. 


\section{الصنف من الدوال متعددة التكافؤ الميرومورفية}

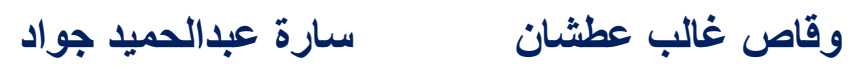

$$
\begin{aligned}
& \text { قسم الرياضيات ـكليه علوم الحاسوب وتكنلوجيان المعلومات } \\
& \text { جامعة القادسية ــ الديوانية ـ العراق }
\end{aligned}
$$

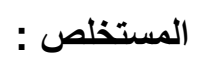

في هذا البحث ،عرفنا الصنف من الدوال متعددة التكافؤ الميرومورفية و درسنا بعض الخصائص مثل حدود المعامل ، مبرهنة الإنغلاق ، حدود التشوية والنمو، النقاط المتطرفة ، نصف قطر التحدب ، التركيب الخطي المحدب و المجاميع الجزئية . 